LA-UR- $11-0 / 524$

Approved for public release;

distribution is unlimited.
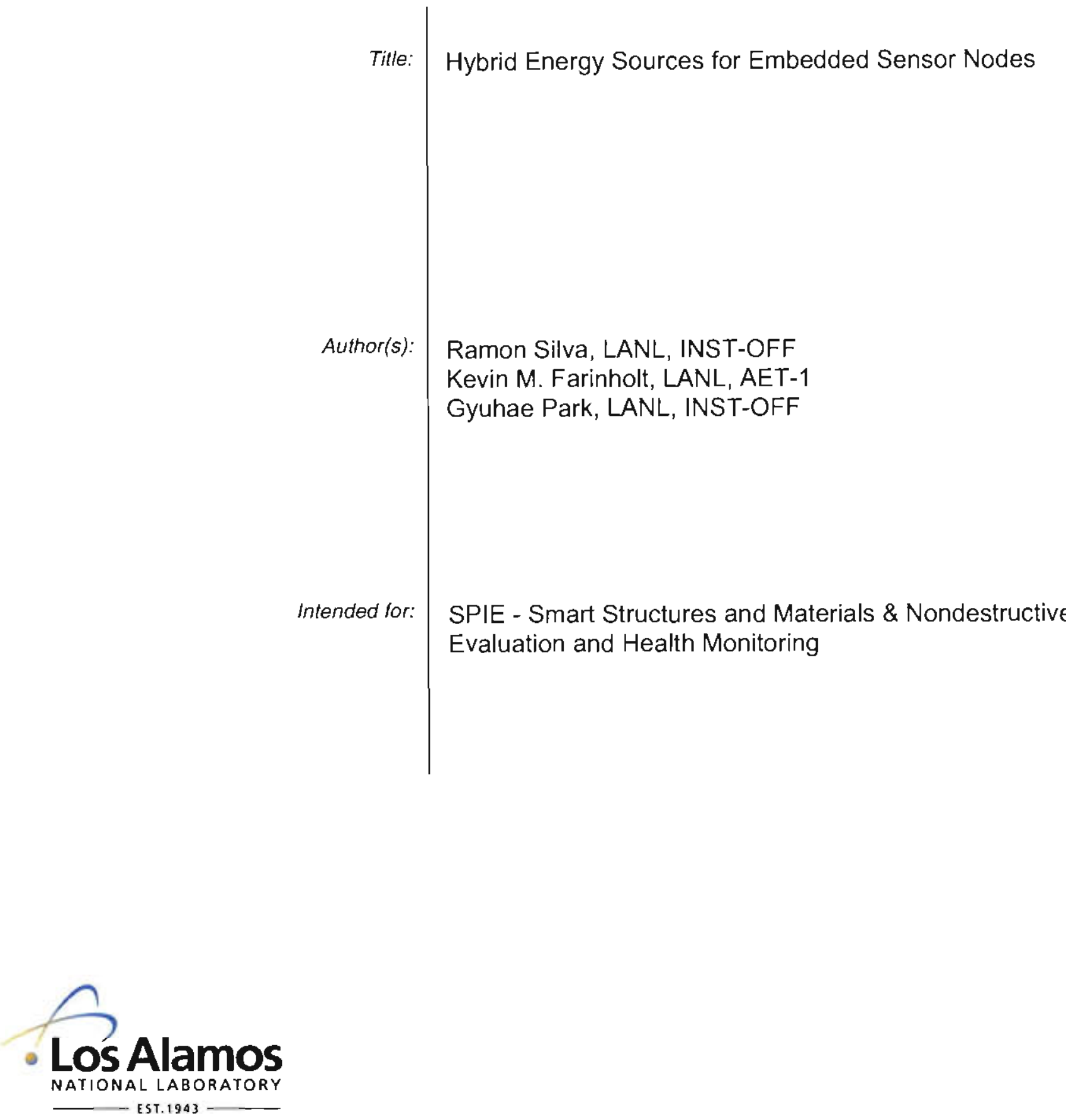

Los Alamos National Laboralory, an affirmative action/equal opportunity employer, is operated by the Los Alamos National Security, LLC for the National Nuclear Security Administration of the U.S. Department of Energy under contract DE-AC52-06NA25396. By acceptance of this article, the publisher recognizes that the U.S. Government retains a nonexclusive, royalty-free license to publish or reproduce the published form of this contribution, or to allow of hers to do so, for U.S. Government purposes. Los Alamos National Laboralory requesis that the publisher identity this article as work performed under the auspices of the U.S. Department of Energy. Los Alamos Nalional Laboratory strongly suppons academic Ireedom and a researcher's right to publish; as an institution, however, the Laboratory does not endorse the viewpoint of a publication or guarantee jts technical correctness.

Form $836(7 / 06)$ 


\section{Hybrid Energy Sources for Embedded Sensor Nodes}

Ramon Silva', Kevin Farinholt'2, Gyuhae Park'

The Engineening Institule, Los Alamos National Laboralory. Los Alamos. NM

?Applied Engineering \& Technology Div., Los Alamos National Laboraton, Los Alamos. NM

March 7-11, 2011

SPIE Sman Structures and Materials +

Nondestructive Evaluation and Heaith Monitoring

$\hat{0}$

A Uncusarme
Abtract
- In this paper, we present a series of hybrid energy configurations that are
designed to provide a robust power source for embedded sensing
hardware. The proper management of energy resources is a critical
component in the design of any deployed sensing network. For systems
that are installed in remote or inaccessible locations, or those with an
operational lifespan that exceeds traditional battery lechnologies, energy
harvesting is an attractive alternative. Unfortunately, the dependence on a
single energy source (i.e. solar) can cause potential problems when
environmental conditions preclude the system from operating at peak
performance. In this paper we consider the use of a hybrid energy source
that extracts energy from multiple sources and uses this collective energy to
power sensing hardware. The sources considered in this work include:
solar, vibration, thermal gradients, and RF energy capture. Methods of
increasing the efficiency, energy storage medium, target apolications and
the integrated use of energy harvesting sources with wireless energy
transmission will be discussed.
- Los Alamos




\section{Presentation Outline}

- Motivation

- Capabilities of the Embedded Sensor Node

- Modes of operation

- Power options

- Energy Harvesting

- Energy Transmission

- Hybrid Powering

- Conclusions

3

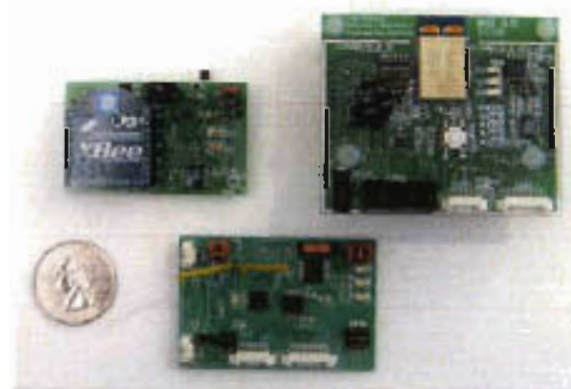

UNCLASSHED

\section{Motivation}

- There has been a recent shift toward wireless sensing systems

- Reduced infrastructure costs over wired systems

- Can retrofit existing civil, industrial or aerospace systems

- Consideration must be given to several design factors

- Sensing capability - active v. passive

- Hardware and software robustness

- Power consumption / powering options

- Systems must be commercially viable

- Can the sensor node be built with COTS components and survive long-term deployment?
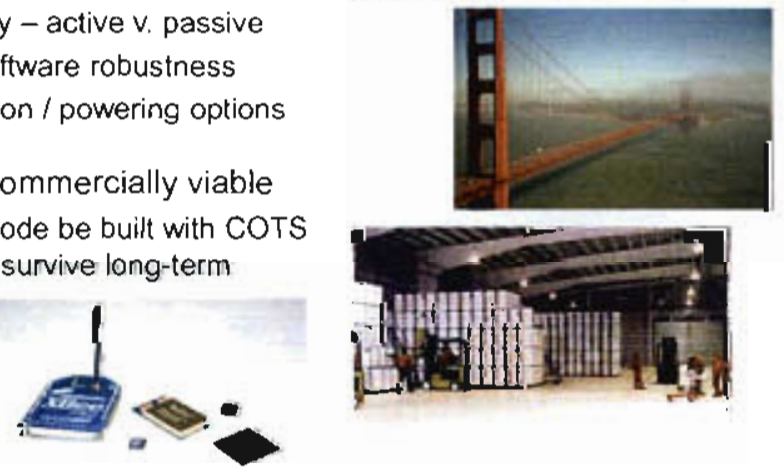


\section{Powering Embedded Hardware}

- Power demands will become a major design criteria for long term deployments

- Currently we are investigating energy harvesting techniques to supplement onboard power sources

- Several sources are available on rotor blades

- Solar, thermal, kinefic, electromagnetic, etc.

- To provide a robust power source, the harvesting system may need to extract energy from multiple sources
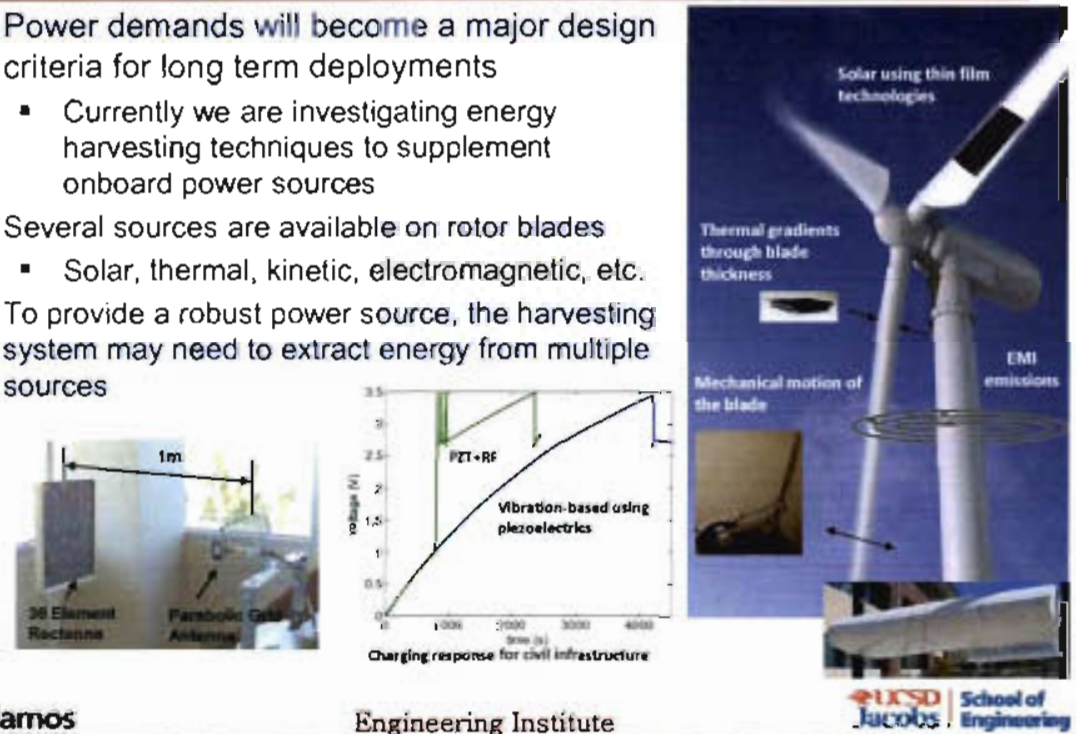

Whas AFIF"

\section{Evolution of the Wireless Impedance Device}

- The first generation WID1 was a breadboard prototype capable of monitoring 1 PZT sensor (Mascarenas / Overly)

- This evolved into a packaged PCB form with the WID1.5

- The WID2 integrated a set of multiplexers, increasing the number of sensors / node to 7, and provides more triggering options (Overly)

- The WID3 builds upon the WID2, providing more stable wireless communication, networking capabilities, and an integrated power conditioning circuit (Taylor)

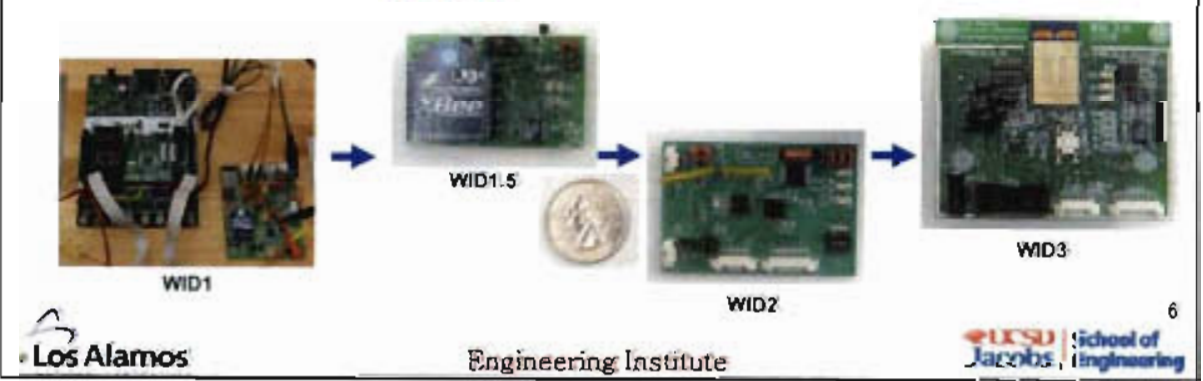



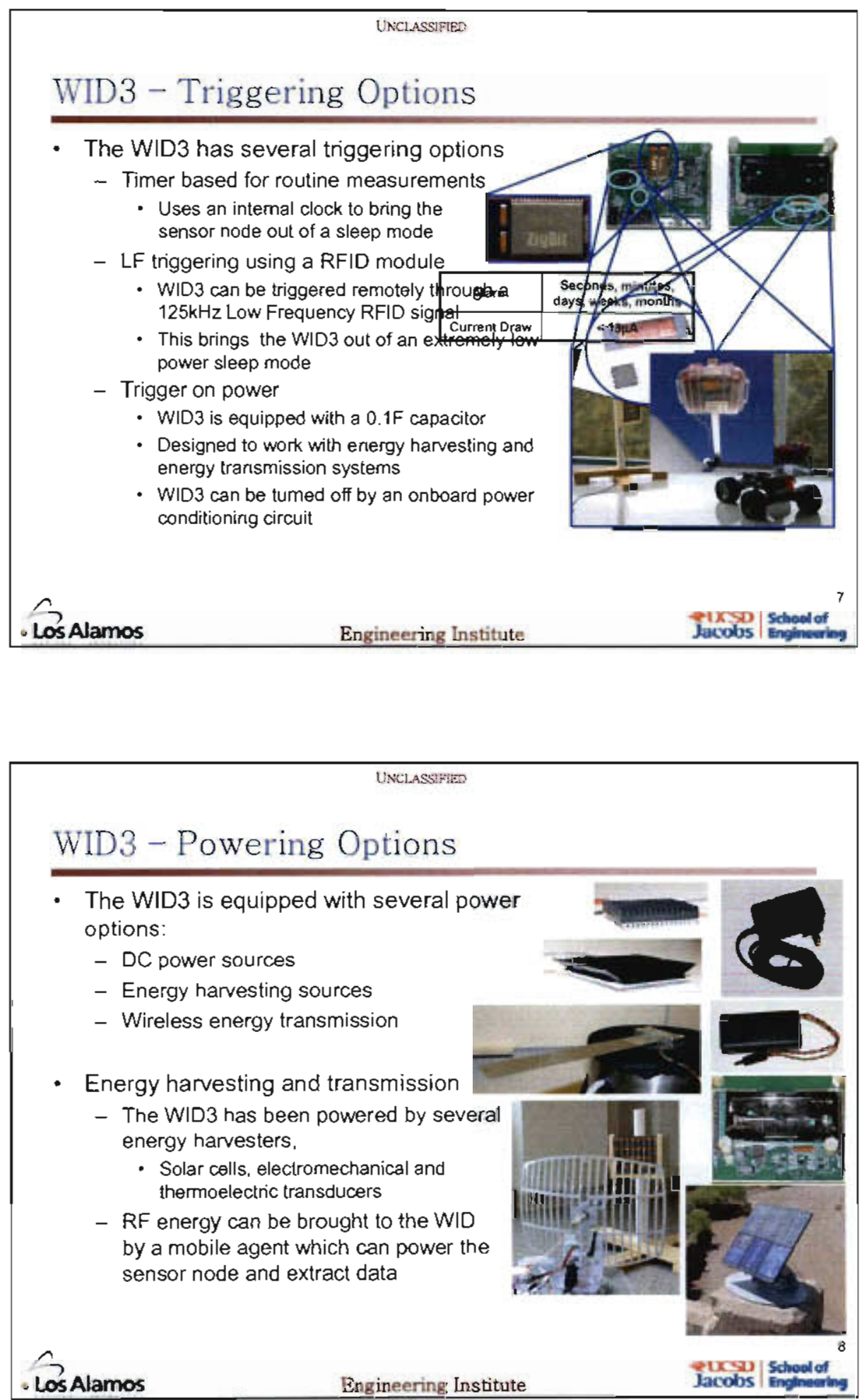

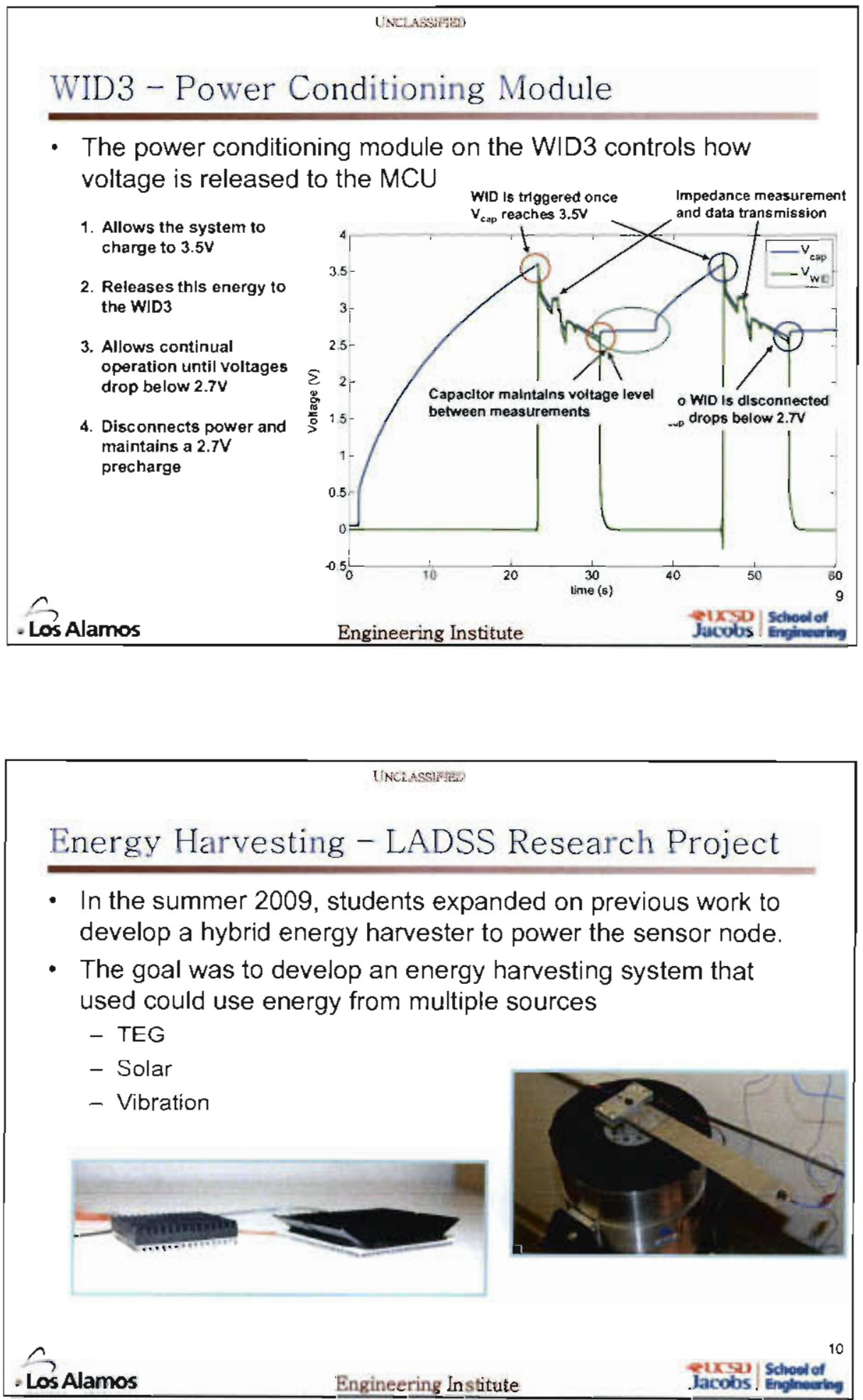


\section{Combined Harvester}

- TEG Module + PZT Beam

- Coupled each system using two ALD EH300A energy harvesting circuits

- TEG module was effective until voltages exceeded $1.2 \mathrm{~V}$

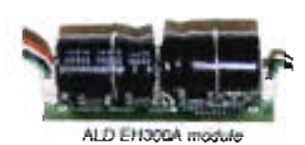

- Successfully powered the WID3 sensor node

- Programmed to measure one sensor and store dala
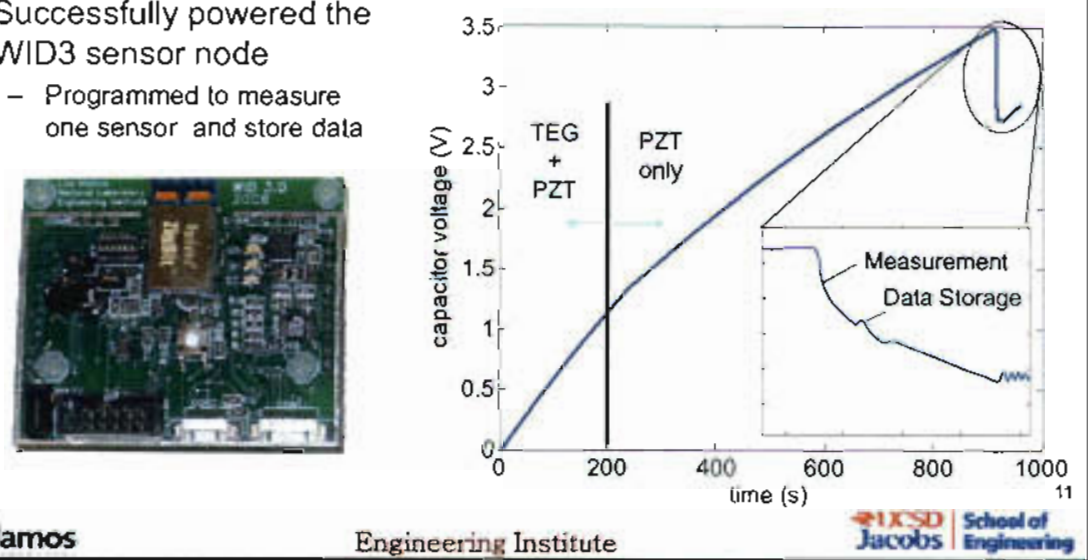

\section{PZT Energy Harvester}

- PSI-5A4E (PZT) piezoceramics from Piezo Systems, Inc.

- Each patch measuring $3.6 \mathrm{~cm} \times 3.6 \mathrm{~cm} \times 0.27 \mathrm{~mm}$

- Mounted in a bimorph configuration

- Cantilevered spring steel substrate $(3.8 \mathrm{~cm} \times 86.5 \mathrm{~cm} \times 0.5 \mathrm{~mm})$

- Two aluminum blocks, $(2.5 \mathrm{~cm})^{3}$, reduced natural frequencies

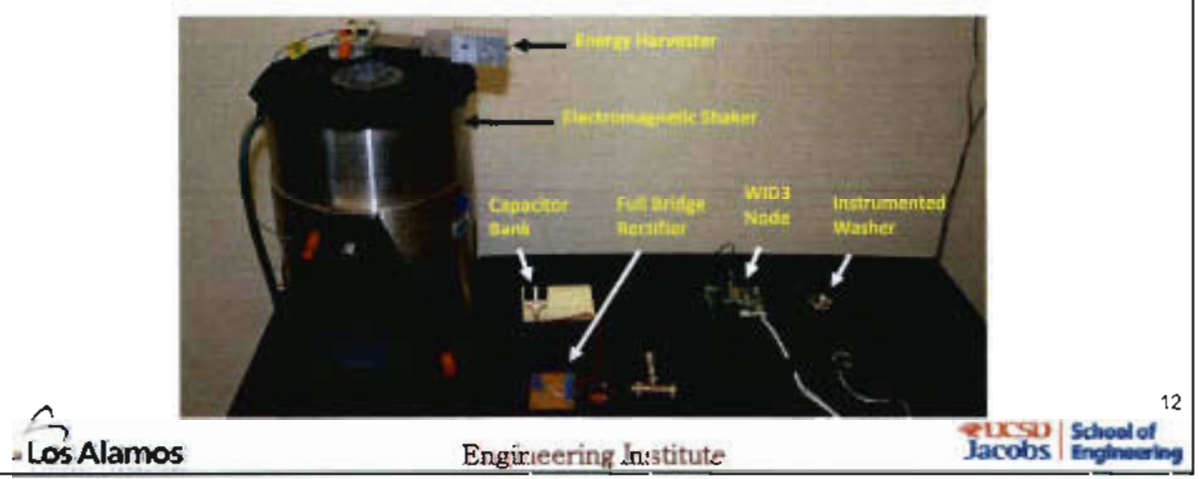




\section{PZT Energy Harvester}

- Harvester was characterized with a $0-1000 \mathrm{~Hz}$ chirp

- First three natural frequencies: $11.8,119.5$, and $775 \mathrm{~Hz}$

- Harvester was excited at its second resonance, $119.5 \mathrm{~Hz}$

- PZT output passed through a full-bridge rectifier

- Rectifier output charged a 0.1F capacitor bank
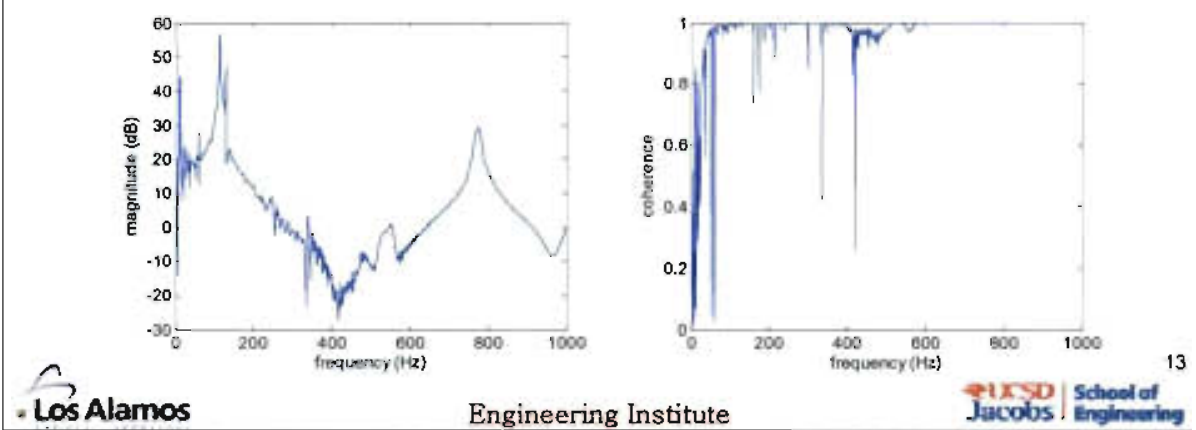

UNCLASSIFIED

\section{PZT Energy Harvester}

- The Harvester required 70 minutes to reach $3.5 \mathrm{~V}$

- WID3 power draw visible in discharge profile - (1) Take measurement, (2) Store data, (3) Confirm storage

- Following voltage drop to $2.7 \mathrm{~V}$, recharging begins
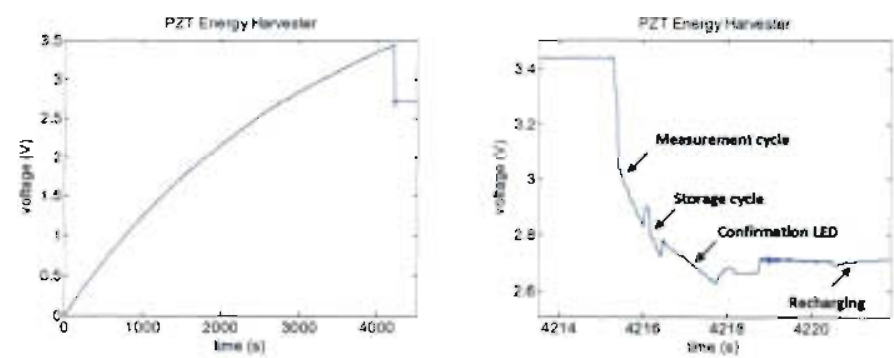


\section{Duty Cycle Limitations}

- Energy harvesting is a viable means of powering an embedded sensor node for long-term deployment

- Total energy levels are low; for most SHM applications, low duty cycles are acceptable

- On-demand measurements may not be possible without a local power source
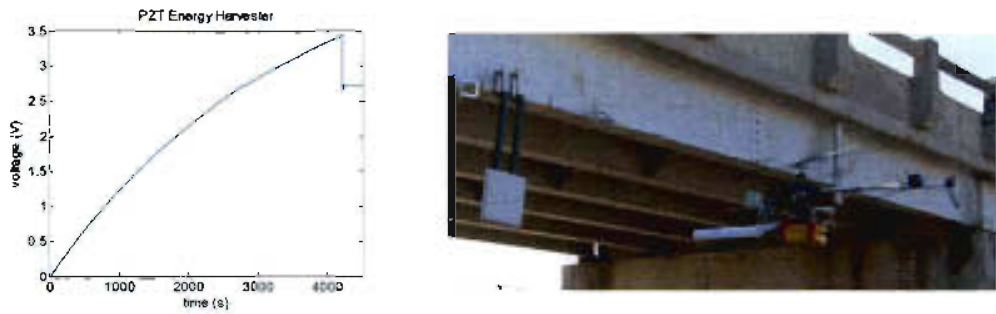

UNCMSSIFED

Wireless Energy Transmission - Supplemental Enerov.

- Energy can be brought to the sensor node at critical times

- RF energy is collected by an antenna connected to the WID3

- This can supplement traditional energy harvesting methods

- Allows off-schedule measurements

- Can be used to power multiple nodes
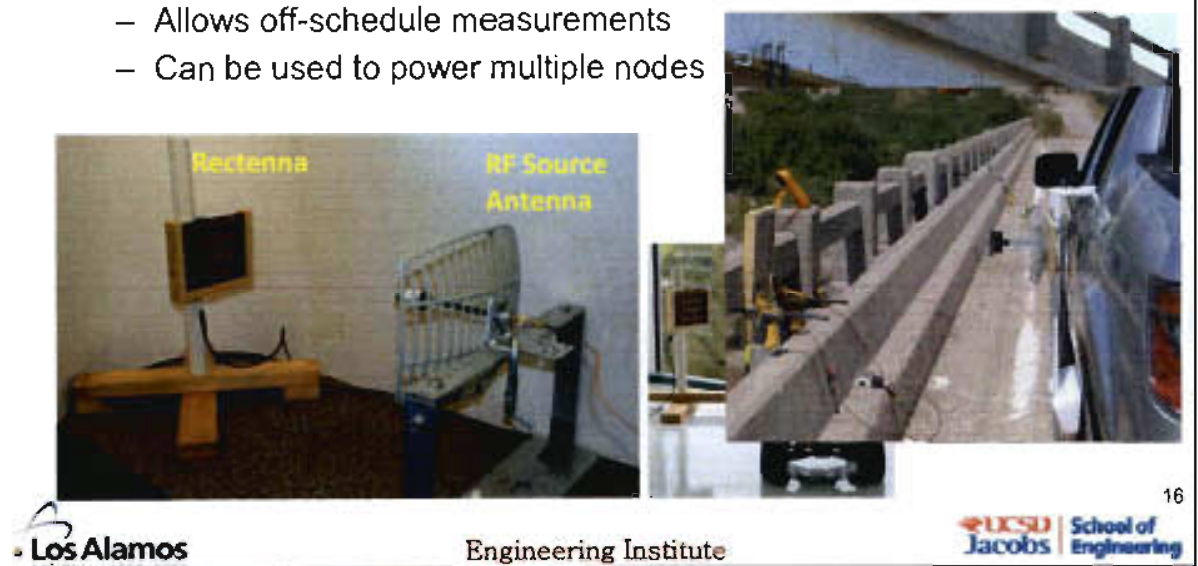


\section{RF Energy Transmission Experiments}

- The sensor node was powered by an RF energy delivery system

- The rectenna array was composed of 32 reclifying antennas

- The Charging time to $3.5 \mathrm{~V}$ was approximately 30 seconds

- The sensor node maintained a $2.7 \mathrm{~V}$ pre-charge between cycles
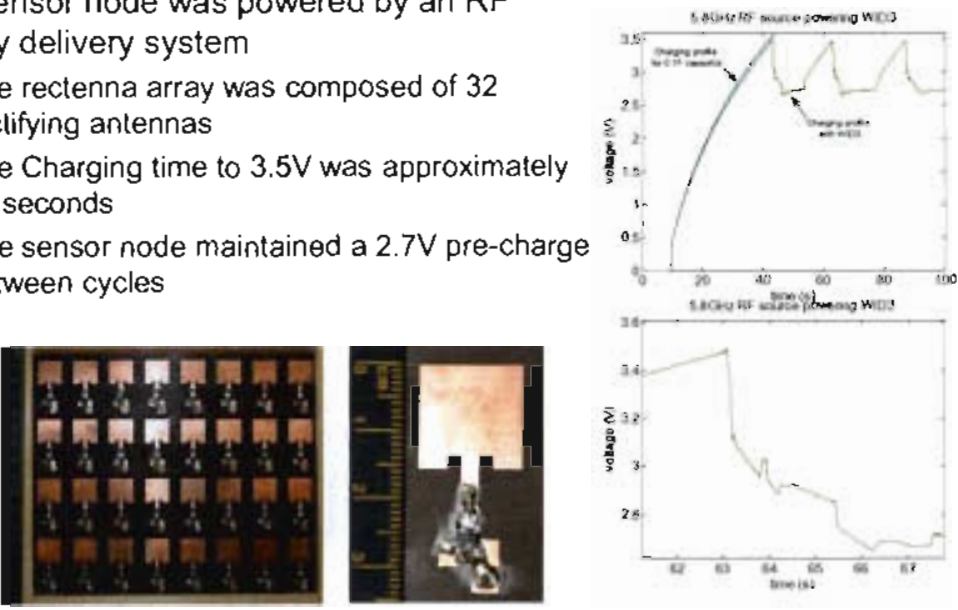

\section{7} Jacobs| Schoel of

I.w口

\section{RF Energy as Power for On-Demand}

\section{Mancuromente}

- At any point in the charge cycle, RF energy can be utilized to quickly provide sufficient energy for multiple measurements

- Following the on-demand measurement, the node can return to its previously scheduled measurement cycle
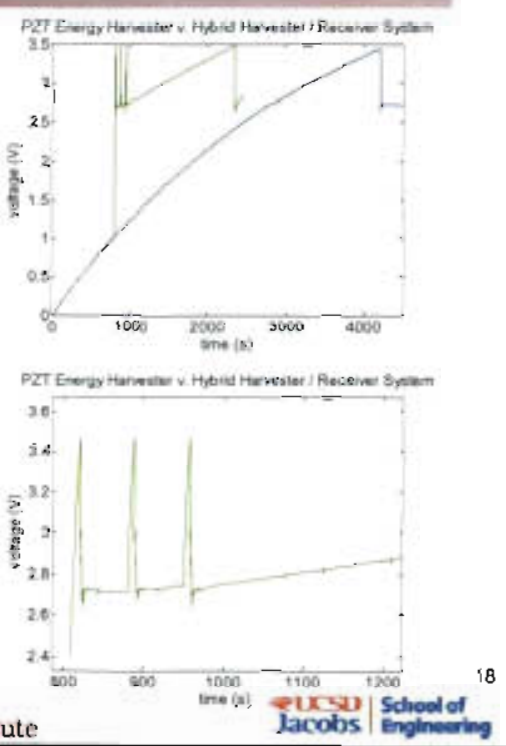


Summary
- The WID provides a compact, power efficient module that can
accept power from numerous sources
- Traditional batteries, energy harvesters, energy lransmission
- Electromechanical and thermoelectric energy harvesters can
successfully generate enough energy to operate the WID
- Provides periodic measurements at low duty cycles
- Radio Frequency (RF) power delivery can sustain continuous on-
demand measurements
The utility of a combined harvester and RF power system has been
demonstrated for adding on-demand capability to scheduled
measurements
Las Alamos

